Pacific Journal of Mathematics

AVERAGING STRONGLY SUBADDITIVE SET FUNCTIONS IN 


\title{
AVERAGING STRONGLY SUBADDITIVE SET FUNCTIONS IN UNIMODULAR AMENABLE GROUPS II
}

\author{
W. R. EMERSON
}

This paper continues work initiated in Part I. The central question is one of characterizing a net $\left\{A_{\alpha}\right\}$ of Borel sets in the group $G$ which averages a so-called regular set function $S$ on $G$ in the sense that $\lambda\left(A_{\alpha}\right)^{-1} S\left(A_{\alpha}\right)$ has a limit (depending only on $S$ ), where $\lambda$ is Haar measure. In Part I a sufficient condition for $\left\{A_{\alpha}\right\}$ to always average was derived; here we show that a "natural" relaxation of this condition is no longer sufficient for all regular $S$, at the same time essentially characterizing those $S$ which may still be averaged. Moreover, the role of Folner summing sequences is considered in this context. Finally, properties of regular set functions are derived which may be of independent interest.

1. Definitions, notation, and some generalities. The notation and terminology is the same as [3]. We also will have frequent cause to refer to the results and techniques of [3], and consequently there is a strong dependence. Throughout the topological group $G$ is assumed locally compact and noncompact and equipped with a fixed right invariant Haar measure $\lambda, \mathscr{K}=\mathscr{K}(G)$ is the set of all precompact Borel subsets of $G, \mathscr{K}_{+}=\{K \in \mathscr{K}: \lambda(K)>0\}, \mathscr{K}_{0}=\{K \in \mathscr{K}: K$ open $\}$, and for $A, K \subseteq G$ we define $[A]_{K}=\{g \in G: K g \subseteq A\}$. Finally as in [3], $\lambda(E)$ denotes inner measure in case $E$ is not measurable, e.g. some $[A]_{K}$ or KA.

Definition 1.1. A net $\left\{A_{\alpha}\right\}$ of sets in $\mathscr{K}_{+}$is said to be:

1. Admissible, $\left\{A_{\alpha}\right\} \in \mathscr{A}$, iff $\lim _{\alpha} \lambda\left(\bar{A}_{\alpha}\right)^{-1} \lambda\left(\left[A_{\alpha}\right]_{K}\right)=1$ for all $K \in \mathscr{K}_{0}$,

2. Full, $\left\{A_{\alpha}\right\} \in \mathscr{F}$, iff $\lim _{\alpha} \lambda\left(A_{\alpha}\right)^{-1} \lambda\left(\left[A_{\alpha}\right]_{K}\right)=1$ for all $K \in \mathscr{K}_{0}$, $K \neq \varnothing$,

3. Stable, $\left\{A_{\alpha}\right\} \in \mathscr{S}$, iff $\lim \lambda\left(K A_{\alpha}\right)^{-1} \lambda\left(A_{\alpha}\right)=1$ for all $K \in \mathscr{K}_{0}$,

4. Translative, $\left\{A_{\alpha}\right\} \in \mathscr{T}$, iff $\lambda\left(A_{\alpha}\right)^{-1} \lambda\left(k A_{\alpha} \Delta A_{\alpha}\right) \rightarrow 0$ uniformly for $k \in K$, for all $K \in \mathscr{K}_{0}$.

Comment. $\mathscr{K}_{0}$ in the above definition may be equivalently replaced by any subfamily of $\mathscr{K}$ which is cofinal under inclusion.

The following result gives some basic properties of these nets, some already proved in [3] and the others straightforward and left to the reader: 
Proposition 1.2. (i) $\mathscr{A} \neq \varnothing$ iff $\mathscr{F} \neq \varnothing$ iff $\mathscr{S} \neq \varnothing$ iff $\mathscr{T} \neq \varnothing$ iff $G$ is unimodular and amenable.

(ii) If $G$ is discrete, $\mathscr{A}=\mathscr{F}=\mathscr{S}=\mathscr{T}$, and otherwise $\mathscr{A} \subset \mathscr{F}$ and $\mathscr{S} \subset \mathscr{T}$ properly and no other inclusions hold.

(iii) $\left\{A_{\alpha}\right\} \in \mathscr{S}(\mathscr{T})$ iff $\left\{A_{\alpha}^{-1}\right\}$ is strong (weak) summing (in the sense of [2]) in unimodular $G$.

(iv) $\left\{A_{\alpha}\right\} \in \mathscr{A}$ and $\left\{B_{\alpha}\right\}$ from $\mathscr{K}$ such that $\lambda\left(\bar{A}_{\alpha}\right)^{-1} \lambda\left(\bar{B}_{\alpha}\right) \rightarrow 0$ implies $\left\{C_{\alpha}\right\} \in \mathscr{A}$ where $C_{\alpha} \doteqdot A_{\alpha} \cup B_{\alpha}$.

(v) $\left\{A_{\alpha}\right\} \in \mathscr{F}$ and $\left\{B_{\alpha}\right\}$ from $\mathscr{K}$ such that $\lambda\left(A_{\alpha}\right)^{-1} \lambda\left(B_{\alpha}\right) \rightarrow 0$ implies $\left\{C_{\alpha}\right\} \in \mathscr{F}$ where $C_{\alpha} \doteqdot A_{\alpha} \cup B_{\alpha}$.

The following definition from [3] is needed:

Definition 1.3. $\mathscr{K}_{c} \doteqdot\{K \in \mathscr{K}: \lambda(K)=\lambda(\bar{K})\}$ is called the class of weak continuity sets in $G$.

Note. Every compact set is a weak continuity set.

We conclude this section with the definition of the set functions $S$ which we shall consider as well as the basic "rearrangement" inequality for such $S$ : iff

Definition 1.4. (a) A set function $S: \mathscr{K} \rightarrow R$ is said to be regular

(i) $\quad S \leqq 0, S(\varnothing)=0$,

(ii) $S(A \cup B)+S(A \cap B) \leqq S(A)+S(B)$ for all $A, B \in \mathscr{K}$,

(iii) $S(A g)=S(A)$ for all $A \in \mathscr{K}, g \in G$.

(b) A set function $S: \mathscr{K} \rightarrow R$ is said to be upper continuous at $K_{0} \in \mathscr{K}$ iff $\left\{K_{n}\right\} \subseteq \mathscr{K}$ and $\lambda\left(K_{0} \Delta K_{n}\right) \rightarrow 0$ implies

$$
\underline{\lim } S\left(K_{n}\right) \leqq S\left(K_{0}\right) .
$$

(c) A set function $S: \mathscr{K} \rightarrow R$ is said to be upper continuous on $\mathscr{K}$ iff $S$ is upper continuous at each $K_{0} \in \mathscr{K}$.

(d) $S: \mathscr{K} \rightarrow R$ is said to be continuous at $\varnothing$ iff $\left\{K_{n}\right\} \subset \mathscr{K}$ such that $K_{n} \supseteq K_{n+1}$ and $\lambda\left(K_{n}\right) \rightarrow 0$ implies $S\left(K_{n}\right) \rightarrow S(\varnothing)$.

Equivalent formulations for regular $S$ are contained in:

PROPOSITION 1.5. (i) The regular set function $S$ is upper continuous at $K_{0}$ iff for every $\epsilon>0$ there corresponds a $\delta=\delta\left(K_{0}, \epsilon\right)>0$ such that $K \in \mathscr{K}, K \subseteq K_{0}$ and $\lambda\left(K_{0}-K\right)<\delta$ implies $S(K)<S\left(K_{0}\right)+\epsilon$.

(ii) The regular set function $S$ is continuous at $\varnothing$ iff given $K \in \mathscr{K}$ and $\epsilon>0$ there corresponds a $\delta=\delta(K, \epsilon)>0$ such that $E \subseteq K, E \in \mathscr{K}$, and $\lambda(E)<\delta$ implies $S(E)>-\epsilon$. 
Proof. If (i) is violated at $K_{0}$ for $\epsilon=\epsilon_{0}>0$ then for $\delta=1 / n$ we may choose $K=K_{n} \subseteq K_{0}$ with $\lambda\left(K_{0}-K_{n}\right)<1 / n$ and $S\left(K_{n}\right) \geqq S\left(K_{0}\right)+\epsilon_{0}$. But clearly $\lambda\left(K_{0} \Delta K_{n}\right) \rightarrow 0$ and $\underline{\lim } S\left(K_{n}\right) \geqq S\left(K_{0}\right)+\epsilon_{0}$ which violates (b). Conversely, if $\lambda\left(K_{0} \Delta K_{n}\right)<\delta$ then $K=K_{n} \cap K_{0} \subseteq K_{0}$ and also $\lambda\left(K_{0}-K\right) \leqq \lambda\left(K_{0} \Delta K_{n}\right)<\delta$ implying $S(K)<S\left(K_{0}\right)+\epsilon$ by (i). But by the monotonicity of $S, S\left(K_{n}\right) \leqq S(K)$ implying $\underline{\lim } S\left(K_{n}\right)<S\left(K_{0}\right)+\epsilon$ (since $\lambda\left(K_{0} \Delta K_{n}\right)<\delta$ for $n$ sufficiently large, where $\delta=\delta(K, \epsilon)$ ) valid for all $\epsilon>0$ and thus $\underline{\lim } S\left(K_{n}\right) \leqq S\left(K_{0}\right)$ and (b) is verified.

The proof that condition (ii) implies (d) is similar ( $K \doteqdot K_{1}, E=K_{n}$ for $n$ sufficiently large). Conversely if (ii) is violated for $K \in \mathscr{K}$ and $\epsilon=\epsilon_{0}>0$, for each integer $n$ there corresponds an $E=E_{n} \subseteq K$, $E_{n} \in \mathscr{K}$, and satisfying $\lambda\left(E_{n}\right)<1 / n^{2}$ whereas $S\left(E_{n}\right) \leqq-\epsilon_{0}$. Set $K_{n}=$ $\cup\left\{E_{j}: j \geqq n\right\}$, implying $S\left(K_{n}\right) \leqq S\left(E_{n}\right) \leqq-\epsilon_{0}, K_{n} \supseteq K_{n+1}$, and $\lambda\left(K_{n}\right) \rightarrow 0$, violating $(\mathrm{d})$ since $S(\varnothing)=0$.

Proposition 1.6. Let $S: \mathscr{K} \rightarrow R$ satisfy $S(K \cup T)+S(K \cap T) \leqq$ $S(K)+S(T)$ for all $K, T \in \mathscr{K}$. Then for any $K, \in \mathscr{K}, 1 \leqq j \leqq n$,

$$
\sum_{j=1}^{n} S\left(I_{j}\right) \leqq \sum_{j=1}^{n} S\left(K_{j}\right)
$$

where

$$
I_{J}=\left\{g \in G: \sum_{i \leqq n} \chi_{K_{i}}(g) \geqq j\right\} .
$$

Proof. Well known, readily shown by induction on $n$ the given condition being the case $n=2$.

2. Full nets and regular set functions $S$. In this section we investigate necessary and sufficient conditions on $S$ to insure that it is "successfully" averaged on full nets. First a notationally convenient definition and a basic result from [3]:

DEFINITION 2.1. For any function $S: \mathscr{K}_{+} \rightarrow R$ let $M_{S}(K) \doteqdot$ $\lambda(K)^{-1} S(K)$, the average of $S$ on $K$, for $K \in \mathscr{K}_{+}$.

THEOREM 2.2. If $S$ is any regular set function on $\mathscr{K}$ and $\left\{A_{\alpha}\right\} \in \mathscr{A}$,

$$
\begin{aligned}
\lim _{\alpha} M_{S}\left(A_{\alpha}\right) & =\inf \left\{M_{S}(K): K \in \mathscr{K}_{c} \cap \mathscr{K}_{+}\right\} \\
& =\inf \left\{M_{S}(K): K \in \mathscr{K}_{c} \cap \mathscr{K}_{0}\right\} .
\end{aligned}
$$

Now since $\mathscr{A} \varsubsetneqq \mathscr{F}$ (for $\mathscr{A} \neq \varnothing$ and $G$ nondiscrete) the question remains as to whether Theorem 2.2 (or some modification) remains true 
for $\left\{A_{\alpha}\right\} \in \mathscr{F}$ without further restriction on $S$. The following example due to Banach [1], after simple reworking, shows this is not the case:

EXAMPLE 2.3. There exists a finitely additive translation invariance measure $m_{0}$ on the Borel sets of the circle - realized as $[0,1)$ - and a Borel subset $N$ of Lebesgue measure zero, $\lambda(N)=0$, for which $m_{0}(N)=$ $m_{0}([0,1))=1$.

COROllary 2.4. There exists a finitely additive translation invariant measure $m$ on $\mathscr{K}=\mathscr{K}(R)$ such that $m(N)=m([0,1))=1$ for a Lebesgue null Borel set $N \subseteq[0,1)$.

Proof. For $A \in \mathscr{K}, \quad$ define $m(A) \doteqdot \Sigma\left\{m_{0}(\{(A+n) \cap[0,1)\})\right.$ : $n \in Z\}, m_{0}$ as in Example 2.3. That $m$ has the desired properties is readily verified, where $N$ is the set referred to in Example 2.3.

Proposition 2.5. There is a regular set function $S$ on $\mathscr{K}=\mathscr{K}(R)$ such that given any $l \leqq-1$ there exists a sequence $\left\{C_{n}\right\}=\left\{C_{n}(l)\right\}$ in $\mathscr{F}$ satisfying $\lim _{n} M_{S}\left(C_{n}\right)=l$. Moreover, it follows that we may also take $l=-\infty$ above, and in general $M_{S}\left(C_{n}\right)$ need not tend to a limit at all for appropriate $\left\{C_{n}\right\} \in \mathscr{F}$.

Proof. Of course $A_{n}=[0, n]$ is a full sequence in $R$. Consequently by Proposition $1.2(\mathrm{v})$ if $B_{n}=B_{n}(l)=\cup\{i+N$ : $i=1,2, \cdots,[-\ln ]\}$, where $[x]$ is the greatest integer $\leqq x$ and $N$ is from Corollary 2.4, $C_{n}=C_{n}(l)=A_{n} \cup B_{n}$ is also a full sequence since $B_{n}$ is a (Haar) null set. Now if $S \doteqdot-m$ where $m$ is from Corollary 2.4 it is immediate that $S\left(C_{n}\right)=-[-\ln ], \lambda\left(C_{n}\right)=n$, and consequently $M_{S}\left(C_{n}\right)=-[-\ln ] / n \rightarrow l$ as asserted. The final sentence of Proposition 2.5 follows easily from the first.

Whether an analogue of Proposition 2.5 is valid in a general amenable, unimodular, nondiscrete $G$ has not been investigated but should follow from known methods of creating translation invariant functionals in amenable groups. At any rate, we see that in general full nets do not "average" a general regular $S$ and some restriction is necessary. Our basic result is the following sufficient condition, and a corollary:

THEOREM 2.6. If $S$ is regular and continuous at $\varnothing$, then for any $\left\{A_{\alpha}\right\} \in \mathscr{F}$ we have

$$
\lim _{\alpha} M_{S}\left(A_{\alpha}\right)=\inf \left\{M_{S}(K): K \in \mathscr{K}_{+}\right\}=\inf \left\{M_{S}(K): K \in \mathscr{K}_{0} \cap \mathscr{K}_{c}\right\}
$$


Corollary 2.7. If $S$ is regular and $\inf \left\{M_{S}(K): K \in \mathscr{K}_{+}\right\}$is finite, then equation (1) above is valid for any $\left\{A_{\alpha}\right\} \in \mathscr{F}$.

The proof of Theorem 2.6 proceeds by a series of lemmas:

LEMMA 2.8. Let $X$ be a set, $\Sigma$ a field of subsets of $X$, and $\mu$ a finitely additive nonnegative measure on $\Sigma$ normalized by $\mu(X)=1$. Then for any $T_{i} \in \Sigma, 1 \leqq i \leqq k$,

(i) there exists a sequence $\left\{e_{n}\right\} \subseteq X$ (not necessarily distinct) such that for $1 \leqq i \leqq k$,

$$
\mu_{N}\left(T_{\imath}\right) \doteqdot N^{-1} \Sigma\left\{\chi_{T_{\imath}}\left(e_{n}\right): n \leqq N\right\} \rightarrow \mu\left(T_{\imath}\right) \text { as } N \rightarrow+\infty .
$$

(ii) Moreover if $\mu$ gives all points measure 0 , the terms $e_{n}$ in (i) may be chosen to all be distinct, and in fact we may find a countably infinite family of such sequences $\left\{e_{n}^{(j)}\right\}$ satisfying (i) with $e_{n}^{(j)}=e_{m}^{(r)}$ iff $n=m$ and $j=r$ and such that $\mu_{N}\left(T_{i}\right)$ is the same when computed on all sequences $\left\{e_{n}^{()}\right\}$.

Comment. The proof is in fact constructive, informally speaking.

Proof. Partition $\cup\left\{T_{i}: 1 \leqq i \leqq k\right\}$ into $2^{k}-1$ disjoint subsets $\cap\left\{C_{l}: 1 \leqq i \leqq k\right\}$ where $C_{l} \in\left\{T_{l}, T_{i}^{\prime}\right\}$ and not all $C_{i}=T_{i}^{\prime}$. Ignoring those subsets which have measure 0 , list the remaining in some order: $E_{1}, \cdots, E_{r}\left(r \leqq 2^{k}-1\right)$. Therefore all $E_{\text {l }}$ are pairwise disjoint, of positive $\mu$ measure, and any $T_{t}$ is the union of an appropriate subfamily of the $E_{j}$ and a set of $\mu$ measure 0 . Now to the $\left\{E_{J}\right\}$ correspond disjoint intervals $I_{1}$ of $[0,1)$ of the same (Lebesgue) measure, e.g. $E_{1} \leftrightarrow\left[0, \mu\left(E_{1}\right)\right)$ and

$$
E_{\jmath} \leftrightarrow\left[\mu\left(\cup\left\{E_{\imath}: i<j\right\}\right), \mu\left(\cup\left\{E_{i}: i \leqq j\right\}\right)\right) \text { for } 1<j \leqq r .
$$

Note that $\mu\left(\cup\left\{E_{l}: 1 \leqq i \leqq r\right\}\right)=\mu\left(\cup\left\{T_{l}: 1 \leqq i \leqq k\right\}\right)$, and consequently if the $I_{1}$ do not cover $[0,1)$ we must have $X-\cup\left\{T_{t}: 1 \leqq i \leqq k\right\}$ of positive $\mu$ measure (and therefore $\neq \varnothing$ ). Now let $\left\{x_{n}\right\}$ be a sequence in $[0,1$ ) uniformly distributed in the classical sense, e.g. the fractional part of $n \alpha$ for any irrational $\alpha$. The sequence $\left\{e_{n}\right\}$ is chosen as follows: if $x_{n} \in I_{j}$ choose $e_{n}$ to be any point in $E_{J}$, and if $x_{n}$ is not in any $I_{j}$ (implying the $I_{j}$ do not cover $[0,1)$ ) choose $e_{n}$ to be any point in $X-\cup\left\{T_{i}: 1 \leqq i \leqq k\right\} \neq \varnothing$. It is immediate that any sequence $\left\{e_{n}\right\}$ chosen in this fashion satisfies (i). Part (ii) follows since any set of positive measure must now contain an infinity of points and consequently may be written as the disjoint union of a countable infinity of infinite sets. Now it is not difficult to see that if the selection of the $\left\{e_{n}\right\}$ as above is done recursively we need not repeat any term, and moreover, by the preceding sentence, in a countable 
infinity of ways with no term used twice in the entire array. Finally, that $\mu_{N}\left(T_{i}\right)$ is independent of the sequence is trivial from the construction since it only depends on the distribution of $\left\{x_{1}, \cdots, x_{N}\right\}$ in $[0,1)$.

LEMMA 2.9. Using the notation of Lemma 2.8, let $\Sigma$ ' be a countable subfamily of $\Sigma$. Then

(i) there exists a sequence $\left\{e_{n}\right\} \subseteq X$ (not necessarily distinct) such that $\mu_{N}(T) \rightarrow \mu(T)$ as $N \rightarrow+\infty$ for all $T \in \Sigma^{\prime}$;

(ii) moreover if $\mu$ gives all points measure 0 , the terms $e_{n}$ in (i) may be chosen to all be distinct.

Comment. The full analog of Lemma 2.8 (ii) is in fact seen to be valid but is of no use here.

Proof. List the sets in $\Sigma^{\prime}$ sequentially as $\left\{T_{i}\right\}$, and by Lemma 2.8 for each $k=1,2, \cdots$. Choose a sequence $\left\{e_{n, k}\right\}$ such that $\mu_{N}\left(T_{i}\right) \rightarrow \mu\left(T_{i}\right)$ for $1 \leqq i \leqq k$ (computed with respect to $\left\{e_{n, k}\right\}$ ). Therefore there is an index $N_{k}$ such that for $1 \leqq i \leqq k$,

$$
\left|\mu\left(T_{i}\right)-N_{k}^{-1} \sum\left\{\chi_{T_{i}}\left(e_{n, k}\right): n \leqq N_{k}\right\}\right|<\frac{1}{k} .
$$

Next let $\left\{w_{k}\right\}$ be any sequence tending to $+\infty$, and let $\left\{M_{k}\right\}$ be any sequence of positive integers chosen such that $\sum_{k<n} M_{k} N_{k} \geqq w_{n} N_{n}$ $(n=2,3, \cdots)$ with $N_{k}$ as in (2). This may be arranged by letting $M_{k}$ tend to $+\infty$ sufficiently rapidly, e.g. if $M_{n-1} \geqq w_{n} N_{n} / N_{n-1}(n=2,3, \cdots)$. Then the desired sequence $\left\{e_{n}\right\}$ is obtained by successively running through the first $N_{1}$ terms of $\left\{e_{n, 1}\right\} \dot{M}_{1}$ times, the first $N_{2}$ terms of $\left\{e_{n, 2}\right\} M_{2}$ times, etc. The verification of (i) is now a simple exercise in Cesaro means whereas (ii) follows from (ii) of Lemma 2.8 upon modifying the construction above appropriately.

Lemma 2.10. Let $K \in \mathscr{K}_{0}$ and $B \in \mathscr{K}$. Then there is a countable subfamily $\mathscr{K}^{\prime} \subseteq \mathscr{K}_{0}$ such that given any $b \in B$, and $\epsilon>0$ there corresponds a $T=T(b, \epsilon) \in \mathscr{K}^{\prime}$ such that $T \subseteq K b$ and $\lambda(K b-T)<\epsilon$.

Proof. Fix a positive integer $n$, and by [3, 1.7 (i)] choose $U=$ $U_{n} \in \mathscr{K}_{0} \cap \mathscr{K}_{c}$ such that $\bar{U} \subseteq K$ and $\lambda(K-U)<1 / n$. As in the proof of $[3,1.5]$, choose $O=O_{n}$ an open symmetric neighborhood of the identity $e$ such that $U O^{2} \subseteq K$. Since $\bar{B} \subseteq O B$ is compact there is a finite cover $\cup\left\{O b_{i}: 1 \leqq i \leqq k\right\}$ of $B, b_{i} \in B$ for $1 \leqq i \leqq k$. Consider the sets $U O b_{1}, \cdots, U O b_{k}$ : for $b \in B, b_{i} \in O b$ iff $b \in O b_{i}$ and consequently $U b \subseteq U O b_{i} \subseteq U O^{2} b \subseteq K b \quad$ implying $\quad \lambda\left(K b-U O b_{i}\right) \leqq \lambda(K b-U b)=$ $\lambda(K-U)<1 / n$, and thus the finite family $U O b_{i}, 1 \leqq i \leqq k$, inner- 
approximates all $K b, b \in B$, to within $1 / n$ in measure. The family obtained by taking all the $U O b_{i}$ for $n=1,2, \cdots$ is the required countable family $\mathscr{K}^{\prime}$.

LEMMA 2.11. Let $\left\{f_{n}\right\}$ be a uniformly bounded sequence of $\mu$ measurable functions on a finite measure space $(X, \mu)$ such that:

(i) $\int f_{n} d \mu=0$, all $n$, (ii) $\underline{\lim }_{n} f_{n} \geqq 0 \mu$-a.e.;

then

(1) $\int\left|f_{n}\right| d \mu \rightarrow 0$, (2) $\left|f_{n}\right| \rightarrow 0$ in measure on $X$.

Proof. Fix $\epsilon>0$ and let $E_{n}=E_{n}(\epsilon)$ be defined by $E_{n}=$ $\left\{x \in X: f_{k}(x)>-\epsilon\right.$ for all $\left.k \geqq n\right\}$. Then since $\lim _{n} f_{n}(x) \geqq 0 \mu-$ a.e. we must have $\mu\left(E_{n}\right) \uparrow \mu(X)$ (or $\mu\left(E_{n}^{\prime}\right) \downarrow 0$ ) by Fatou. Also if $X_{n}^{-} \doteqdot$ $\left\{x \in X: f_{\mathrm{n}}(x)<0\right\}$ we obtain

$$
0 \geqq \int_{X_{n}^{-}} f_{n} d \mu \geqq-\epsilon \mu\left(E_{n}\right)-M \mu\left(E_{n}^{\prime}\right)
$$

where $M$ is a uniform bound for all $\left|f_{n}\right|$. Consequently $\underline{\lim }_{n} \int_{X_{n}^{-}} f_{n} d \mu \geqq$ $-\epsilon \mu(X)$ is valid for all $\epsilon>0$, and therefore $\underline{\lim }_{n} \int_{X_{n}^{-}} f_{n} d \mu \geqq 0$. But this implies $\int_{X_{n}^{-}} f_{n} d \mu \rightarrow 0$ and also $\int_{X_{n}^{+}} f_{n} d \mu=-\int_{X_{n}^{-}} f_{n} d \mu \rightarrow 0$ and thus $\int_{X}\left|f_{n}\right| d \mu \rightarrow 0$. Of course (2) is an immediate consequence of (1).

Lemma 2.12. Let $K \in \mathscr{K}_{0}$ and $A \in \mathscr{K}_{+}$. Then there is a sequence $\left\{e_{n}\right\} \subseteq A$ such that if for $T \subseteq A$ we set

$$
\mu_{N}(T) \doteqdot N^{-1} \sum\left\{\chi_{T}\left(e_{n}\right): n \leqq N\right\} \quad \text { and } \quad g_{N}(b)=\mu_{N}\left(K^{-1} b \cap A\right)
$$

then

$$
g_{N}(b) \rightarrow \lambda(A)^{-1} \lambda\left(K^{-1} b \cap A\right) \text { in measure on } K A \text {. }
$$

Proof. Upon applying Lemma 2.10 with $K$ replaced by $K^{-1}$ and $B$ by $K A$ we obtain a countable $\mathscr{K}^{\prime} \subseteq \mathscr{K}_{0}$ such that given any $b \in K A$ and $\epsilon>0$ there is a set $T \in \mathscr{K}^{\prime}$ such that $T \subseteq K^{-1} b$ and $\lambda\left(K^{-1} b-T\right)<\epsilon$. Now set $\Sigma^{\prime}=\left\{T \cap A: T \in \mathscr{K}^{\prime}\right\}$. Then since $\lambda\left(\left(K^{-1} b \cap A\right)-T \cap A\right)=$ $\lambda\left(\left(K^{-1} b-T\right) \cap A\right) \leqq \lambda\left(K^{-1} b-T\right)<\epsilon$, we see that the countable family $\Sigma^{\prime}$ approximates $\left\{K^{-1} b \cap A: b \in K A\right\}$ from within arbitrarily closely in measure. Now apply Lemma 2.9 (with $\mu=\lambda(A)^{-1} \lambda, X=A, \Sigma=\{K \cap$ $A: K \in \mathscr{K}\})$ and let $\left\{e_{n}\right\} \subseteq A$ be a sequence such that $\mu_{N}(T) \rightarrow \mu(T)=$ 
$\lambda(A)^{-1} \lambda(T)$ for all $T \in \Sigma^{\prime}$. Since $g_{N}(b)=\mu_{N}\left(K^{-1} b \cap A\right) \geqq \mu_{N}(T)$ for $T \subseteq K^{-1} b \cap A$, the inner approximating property of $\Sigma^{\prime}$ implies $\underline{\lim }_{N} g_{N}(b) \geqq \lambda(A)^{-1} \lambda\left(K^{-1} b \cap A\right)$ for all $b$ in $K A$. Now set $f_{N}(b) \doteqdot$ $g_{N}(b)-\lambda(A)^{-1} \lambda\left(K^{-1} b \cap A\right)$, and apply Lemma 2.11 (with $X=K A$, $\mu=\lambda \mid K A)$ : first trivially $\left|f_{N}\right| \leqq 1$, and hypothesis (ii) has just been verified above. It remains to verify (i):

$$
\begin{aligned}
& \int_{K A} f_{N}(b) d \lambda(b)=\int_{K A} g_{N}(b) d \lambda(b)-\int_{K A} \lambda(A)^{-1} \lambda\left(K^{-1} b \cap A\right) d \lambda(b) \\
& =N^{-1} \sum_{n \leqq N} \int_{K A} \chi_{K^{-1} b \cap A}\left(e_{n}\right) d \lambda(b)-\lambda(A)^{-1} \int_{K A}\left\{\int_{A} \chi_{K^{-1} b}(a) d \lambda(a)\right\} d \lambda(b) \\
& =N^{-1} \sum_{n \leqq N} \int_{K A} \chi_{K e_{n}}(b) d \lambda(b)-\lambda(A)^{-1} \int_{A}\left\{\int_{K A} \chi_{K a}(b) d \lambda(b)\right\} d \lambda(a)
\end{aligned}
$$

(since $e_{n} \in K^{-1} b \cap A$ iff $b \in K e_{n}$ and $a \in K^{-1} b$ iff $b \in K a$ )

$$
\begin{aligned}
& =N^{-1} \sum_{n \leq N} \lambda\left(K e_{n} \cap K A\right)-\lambda(A)^{-1} \int_{A} \lambda(K a \cap K A) d \lambda(a) \\
& =N^{-1} \sum_{n \leqq N} \lambda\left(K e_{n}\right)-\lambda(A)^{-1} \int_{A} \lambda(K a) d \lambda(a)=\lambda(K)-\lambda(K)=0 .
\end{aligned}
$$

We now conclude that $\left|f_{N}\right| \rightarrow 0$ in measure on $K A$, which is the assertion of Lemma 2.12.

We are now in a position to prove the following strengthened form of $[3,2.3]$.

Proposition 2.13 (The Fundamental Inequality, Strong Form): If $A \in \mathscr{K}_{+}$and $K \in \mathscr{K}_{0}$ and $S$ is continuous at $\varnothing$, then

$$
\lambda(A)^{-1} S(K A) \leqq \lambda\left(K^{-1}\right)^{-1} S(K)
$$

Proof. Fix $K \in \mathscr{K}_{0}$ and $A \in \mathscr{K}_{+}$and let $\left\{e_{n}\right\} \subseteq A$ be the sequence described in Lemma 2.12. We consider

$$
N^{-1} \sum_{j \leqq N} S\left(K e_{j}\right)=S(K)
$$

by Proposition 1.6, since $\cup\left\{K e_{j}: j \leqq N\right\} \subseteq K A$,

$$
N^{-1} \sum_{j \leqq N} S\left(I_{j}\right) \leqq S(K)
$$


where

$$
\begin{aligned}
I_{j}=I_{j}(N) & =\left\{b \in K A: \sum_{n \leqq N} \chi_{K e_{n}}(b) \geqq j\right\} \\
& =\left\{b \in K A: \sum_{n \leqq N} \chi_{K^{-1} b \cap A}\left(e_{n}\right) \geqq j\right\} \\
& =\left\{b \in K A: g_{N}(b) \geqq j / N\right\},
\end{aligned}
$$

using the notation of Lemma 2.12. But by the same Lemma, $g_{N}(b) \rightarrow \lambda(A)^{-1} \lambda\left(K^{-1} b \cap A\right) \leqq \lambda(A)^{-1} \lambda\left(K^{-1}\right)$ in measure on $K A$, and consequently for any $\epsilon>0$ we must have

$$
\lambda\left(\left\{b \in K A: g_{N}(b) \geqq \lambda(A)^{-1} \lambda\left(K^{-1}\right)+\epsilon\right\}\right) \rightarrow 0 \quad \text { as } \quad N \rightarrow+\infty,
$$

i.e. for $j / N \geqq \lambda(A)^{-1} \lambda\left(K^{-1}\right)+\epsilon$, we have $\lambda\left(I_{j}\right) \rightarrow 0$ as $N \rightarrow+\infty$ (uniformly for $j \geqq N\left(\lambda(A)^{-1} \lambda\left(K^{-1}\right)+\epsilon\right)$. Now write

$$
\begin{aligned}
N^{-1} \sum_{j \leqq N} S\left(I_{j}\right)= & N^{-1} \sum\left\{S\left(I_{l}\right): j / N<\lambda(A)^{-1} \lambda\left(K^{-1}\right)+\epsilon, j \leqq N\right\} \\
& +N^{-1} \sum\left\{S\left(I_{l}\right): j / N \geqq \lambda(A)^{-1} \lambda\left(K^{-1}\right)+\epsilon, j \leqq N\right\} .
\end{aligned}
$$

Since $I, \subseteq K A$ always and consequently $S(K A) \leqq S\left(I_{j}\right)$ always, the first term on the right is bounded below by $N^{-1}\left[N\left(\lambda(A)^{-1} \lambda\left(K^{-1}\right)+\epsilon\right)\right] S(K A)$ where $[X]$ is the greatest integer less than $X$. On the other hand, since the $I_{j}$ are nested the second sum is trivially bounded below by $S\left(I_{j}\right)$ where $j=\left[N\left(\lambda(A)^{-1} \lambda\left(K^{-1}\right)+\epsilon\right)\right]$. Therefore

$$
N^{-1}\left[N\left(\lambda(A)^{-1} \lambda\left(K^{-1}\right)+\epsilon\right] S(K A)+S\left(I_{\left[N\left(\lambda(A)^{-1} \lambda\left(K^{-1}\right)+\epsilon\right)\right]}\right) \leqq S(K)\right.
$$

for all positive integers $N$ and $\epsilon>0$. But for fixed $\epsilon>0$ upon letting $N \rightarrow+\infty$ in (4) we obtain

$$
\left(\lambda(A)^{-1} \lambda\left(K^{-1}\right)+\epsilon\right) S(K A) \leqq S(K)
$$

since $S$ is continuous at $\varnothing$ and $\lambda\left(I_{\left[N\left(\lambda(A)^{-1} \lambda\left(K^{-1}\right)+\epsilon\right)\right]}\right) \rightarrow 0$ by (3). The proposition follows upon letting $\epsilon \downarrow 0$.

Theorem 2.6 follows immediately from Proposition 2.13 since an analogue of $[3,2.4]$ may now be proved with $\mathscr{K}_{0}$ instead of $\mathscr{K}_{c} \cap \mathscr{K}_{0}$ in the last inequality. Corollary 2.7 is immediate since $\inf \left\{M_{S}(K): K \in \mathscr{K}_{+}\right\}$ finite trivially implies $S$ must be continuous at $\varnothing$. Note in particular that the Corollary shows that if $\inf \left\{M_{s}(K): K \in \mathscr{K}_{+}\right\}$is finite then so is $\inf \left\{M_{s}(K): K \in \mathscr{K}_{0} \cap \mathscr{K}_{c}\right\}$ (this much is trivial) and they are equal in case $\mathscr{F} \neq \varnothing$, i.e. if $G$ is unimodular and amenable. 
Moreover, we have the following partial converse to Theorem 2.6:

THEOREM 2.14. If $S$ is regular and for all $\left\{A_{\alpha}\right\} \in \mathscr{F} \neq \varnothing$ $\lim _{\alpha} M_{S}\left(A_{\alpha}\right)$ exists (possibly depending on $\left\{A_{\alpha}\right\}$ ), then the limit is in fact independent of $\left\{A_{\alpha}\right\} \in \mathscr{F}$ and equals $\inf \left\{M_{S}(K): K \in \mathscr{K}_{+}\right\}=$ $\inf \left\{M_{S}(K): K \in \mathscr{K}_{0} \cap \mathscr{K}_{c}\right\}$. Moreover (assuming $\mathscr{F} \neq \varnothing$ ) this is the case iff

(i) $\inf \left\{M_{s}(K): K \in \mathscr{K}_{0} \cap \mathscr{K}_{c}\right\}=-\infty$ or

(ii) $S$ is continuous at $\varnothing$.

Proof. From the comment immediately preceding the statement of Theorem 2.14, we see that

$$
\inf \left\{M_{S}(K): K \in \mathscr{K}_{+}\right\}<\inf \left\{M_{S}(K): K \in \mathscr{K}_{0} \cap \mathscr{K}_{c}\right\}
$$

iff the former is $-\infty$ and the latter is finite. If this is the case let $\left\{K_{n}\right\} \subseteq \mathscr{K}_{+}$be chosen such that $M_{S}\left(K_{n}\right)=\lambda\left(K_{n}\right)^{-1} S\left(K_{n}\right) \rightarrow-\infty$. Next, fix any $\left\{A_{\alpha}\right\} \in \mathscr{A} \subseteq \mathscr{F}$, implying $\lim _{\alpha} M_{S}\left(A_{\alpha}\right)=\inf \left\{M_{S}(K): K \in \mathscr{K}_{0} \cap \mathscr{K}_{c}\right\}$ $(>-\infty)$ by Theorem 2.2. Now since $\left[A_{\alpha}\right]_{K} \neq \varnothing$ implies $\lambda\left(A_{\alpha}\right) \geqq \lambda(K)$ it follows that $\lim _{\alpha} \lambda\left(A_{\alpha}\right)=+\infty$. We now wish to expand $\left\{A_{\alpha}\right\}$ à-la Proposition $1.2(\mathrm{v})$ by $\left\{B_{\alpha}\right\}$ where each $B_{\alpha}$ is an appropriately chosen disjoint union of right translates of a set $K_{n}$ for "large" $n$ while $\lambda\left(B_{\alpha}\right)$ is of smaller order than $\lambda\left(A_{\alpha}\right.$, whereas $S\left(B_{\alpha}\right)$ is of larger order than $S\left(A_{\alpha}\right)$. The technical details follow, where for simplicity and without loss of generality we assume $M_{S}\left(K_{n}\right)<-n$ and $\lambda\left(K_{n}\right) \uparrow+\infty$ (if $K g_{i}, 1 \leqq i \leqq r$, are disjoint

$$
\begin{aligned}
& M_{S}\left(\cup\left\{K g_{i}: 1 \leqq i \leqq r\right\}\right)=(r \lambda(K))^{-1} S\left(\cup\left\{K g_{i}: 1 \leqq i \leqq r\right\}\right) \\
& \left.\quad \leqq(r \lambda(K))^{-1}\left(\sum\left\{S\left(K g_{i}\right): 1 \leqq i \leqq r\right\}\right)=\lambda(K)^{-1} S(K)=M_{S}(K)\right)
\end{aligned}
$$

Now consider the real sequence $\left\{2 n^{3 / 2} \lambda\left(K_{n}\right)\right\}$ which tends monotonically to $+\infty$, and choose $\alpha_{0}$ such that $\alpha>\alpha_{0}$ implies $\lambda\left(A_{\alpha}\right) \geqq 2 \lambda\left(K_{1}\right)$. Then for each $\alpha>\alpha_{0}$ define the positive integer $n=n(\alpha)$ by

$$
2 n^{3 / 2} \lambda\left(K_{n}\right) \leqq \lambda\left(A_{\alpha}\right)<2(n+1)^{3 / 2} \lambda\left(K_{n+1}\right)
$$

and note in particular that $\lim _{\alpha} n(\alpha)=+\infty$ since $\lim _{\alpha} \lambda\left(A_{\alpha}\right)=+\infty$. For $\alpha>\alpha_{0}$ then choose $B_{\alpha}$ to consist of $N_{\alpha}$ disjoint right translates of $K_{n}$ where $N_{\alpha}=\left[\lambda\left(A_{\alpha}\right) / n^{1 / 2} \lambda\left(K_{n}\right)\right] \geqq 2 n \geqq 2$. For completeness take $B_{\alpha}=\varnothing$ for all other $\alpha$. Consequently, for $\alpha>\alpha_{0}$,

$$
\begin{aligned}
\lambda\left(B_{\alpha}\right) & =N_{\alpha} \lambda\left(K_{n}\right)=\left[\lambda\left(A_{\alpha}\right) / n^{1 / 2} \lambda\left(K_{n}\right)\right] \lambda\left(K_{n}\right) \\
& \leqq\left(\lambda\left(A_{\alpha}\right) / n^{1 / 2} \lambda\left(K_{n}\right)\right) \lambda\left(K_{n}\right)=\lambda\left(A_{\alpha}\right) / n^{1 / 2}
\end{aligned}
$$


and therefore $\lim _{\alpha} \lambda\left(A_{\alpha}\right)^{-1} \lambda\left(B_{\alpha}\right)=0$. Thus by Proposition $1.2(\mathrm{v})$ we have $\left\{C_{\alpha}\right\} \in \mathscr{F}$ where $C_{\alpha}=A_{\alpha} \cup B_{\alpha}$ for all $\alpha$. Also by the subadditivity of $S$, for $\alpha>\alpha_{0}$

$$
S\left(B_{\alpha}\right) \leqq N_{\alpha} S\left(K_{n}\right) \leqq \frac{1}{2}\left(\lambda\left(A_{\alpha}\right) / n^{1 / 2}\right)\left(\lambda\left(K_{n}\right)^{-1} S\left(K_{n}\right)\right),
$$

since $[x] \geqq \frac{1}{2} x$ for $[x] \geqq 2$, and consequently

$$
\begin{aligned}
M_{S}\left(C_{\alpha}\right) & =\lambda\left(C_{\alpha}\right)^{-1} S\left(C_{\alpha}\right) \leqq \lambda\left(C_{\alpha}\right)^{-1} S\left(B_{\alpha}\right) \\
& =\left(\lambda\left(C_{\alpha}\right)^{-1} \lambda\left(A_{\alpha}\right)\right)\left(\lambda\left(A_{\alpha}\right)^{-1} S\left(B_{\alpha}\right)\right) \\
& \leqq\left(\lambda\left(C_{\alpha}\right)^{-1} \lambda\left(A_{\alpha}\right)\right)\left(M_{S}\left(K_{n}\right) / 2 n^{1 / 2}\right)<\lambda\left(C_{\alpha}\right)^{-1} \lambda\left(A_{\alpha}\right)\left(-n^{1 / 2} / 2\right),
\end{aligned}
$$

since $M_{s}\left(K_{n}\right)<-n$. Therefore $\lim M_{s}\left(C_{\alpha}\right)=-\infty$ since $\lambda\left(C_{\alpha}\right)^{-1} \lambda\left(A_{\alpha}\right)$ $\rightarrow 1$ and $n=n(\alpha) \rightarrow+\infty$ as $\alpha$ "gets large". It is now easy to combine $\left\{A_{\alpha}\right\}$ and $\left\{C_{\alpha}\right\}$ into a single net in $\mathscr{F}$ on which $M_{S}$ does not converge by stipulating that $A_{\alpha}$ (or $C_{\alpha}$ ) is "further out" than $C_{\alpha^{\prime}}\left(\right.$ and $A_{\alpha^{\prime}}$ ) iff $\alpha>\alpha^{\prime}$.

We have shown that if inf $M_{S}$ is different on $\mathscr{K}_{+}$and $\mathscr{K}_{0} \cap \mathscr{K}_{c}$ then $\lim _{\alpha} M_{S}\left(A_{\alpha}\right)$ does not necessarily exist for $\left\{A_{\alpha}\right\} \in \mathscr{F}$. But conversely, by $[3,2.4]$, if they are equal then $\lim _{\alpha} M_{S}\left(A_{\alpha}\right)$ always equals this common value for $\left\{A_{\alpha}\right\} \in \mathscr{F}$. Thus $\lim _{\alpha} M_{S}\left(A_{\alpha}\right)$ exists for all $\left\{A_{\alpha}\right\} \in \mathscr{F}$ iff inf $M_{S}$ is equal on $\mathscr{K}_{+}$and $\mathscr{K}_{0} \cap \mathscr{K}_{c}$, and in this case $\lim _{\alpha} M_{S}\left(A_{\alpha}\right)$ always equals the common value.

The last assertion is now also clear since (i) or (ii) imply inf $M_{S}$ is the same on $\mathscr{K}_{+}$and $\mathscr{K}_{0} \cap \mathscr{K}_{c}$ (see Theorem 2.6 for (ii)). Conversely, if the common value is $-\infty$ then (i) is true whereas if they are both finite and equal (ii) is trivially true.

We conclude this section with the following:

\section{PROPOSITION 2.15.}

1. (strong converse to Proposition 2.13): If $\lambda\left(A_{0}\right)^{-1} S\left(K A_{0}\right) \leqq$ $\lambda\left(K^{-1}\right)^{-1} S(K)$ for all $K \in \mathscr{K}_{0}$ and one $A_{0} \in \mathscr{K}_{+}$then $S$ is continuous at $\varnothing$.

2. $S$ is continuous at $\varnothing$ iff $\left\{K_{n}\right\} \subseteq \mathscr{K}_{+}$and $K_{n} \supseteq K_{n+1}$ always implies $\inf M_{S}\left(K_{n}\right)>-\infty$.

Comment. The second assertion essentially shows that $S$ is continuous at $\varnothing$ iff a "Lipschitz condition" holds at $\varnothing$.

Proof. 1. Choose $\left\{K_{n}\right\} \subset \mathscr{K}$ such that $K_{n} \supseteq K_{n+1}$ and $\lambda\left(K_{n}\right) \rightarrow 0$. By the regularity of $\lambda$ we may find $\left\{U_{n}\right\} \subset \mathscr{K}_{0}$ such that $K_{n} \subseteq U_{n}$ and $\lambda\left(U_{n}-K_{n}\right) \rightarrow 0$. We may assume $U_{n} \supseteq U_{n+1}$ also by considering $U_{n}^{*}=$ $\cap\left\{U_{k}: k \leqq n\right\}$ if necessary. Clearly $\lambda\left(U_{n}\right) \rightarrow 0$ and $S\left(U_{n}\right) \leqq S\left(K_{n}\right)$, so if we initially assume $\left\{K_{n}\right\} \subset \mathscr{K}_{0}$ and show $S\left(K_{n}\right) \rightarrow S(\varnothing)=0$ then the general case follows. Upon taking $K=K_{n}$ in the inequality, 


$$
\lambda\left(A_{0}\right)^{-1} S\left(K_{1} A_{0}\right) \leqq \lambda\left(A_{0}\right)^{-1} S\left(K_{n} A_{0}\right) \leqq \lambda\left(K_{n}^{-1}\right)^{-1} S\left(K_{n}\right)
$$

since $K_{n} \subseteq K_{1}$ and $S$ is monotonic. Since $K_{n} \subseteq K_{1} \subseteq \bar{K}_{1}$ (compact), the modular function $\Delta$ is bounded away from 0 and $+\infty$ on $K_{n}$ uniformly in $n$ and consequently

$$
\lambda\left(K_{n}^{-1}\right)^{-1} \geqq C \lambda\left(K_{n}\right)^{-1}
$$

for an appropriate $C>0$ independent of $n$. Consequently (since $S\left(K_{n}\right)$ $\leqq 0)$

$$
\lambda\left(A_{0}\right)^{-1} S\left(K_{1} A_{0}\right) \leqq C \lambda\left(K_{n}\right)^{-1} S\left(K_{n}\right),
$$

or

$$
\lambda\left(A_{0}\right)^{-1} S\left(K_{1} A_{0}\right) \lambda\left(K_{n}\right) / C \leqq S\left(K_{n}\right) \leqq 0 .
$$

But this clearly implies $S\left(K_{n}\right) \rightarrow 0$ since $\lambda\left(K_{n}\right) \rightarrow 0$ with $n$ (and the other terms on the left are independent of $n)$ and we are done.

2. Assume $S$ is continuous at $\varnothing$ and $\left\{K_{n}\right\} \subseteq \mathscr{K}_{+}$with $K_{n} \supseteq$ $K_{n+1}$. As in the proof of 1 take $\left\{U_{n}\right\} \subseteq \mathscr{K}_{0}$ with $U_{n} \supseteq K_{n}$ and $\lambda\left(U_{n}\right)<$ $2 \lambda\left(K_{n}\right)$ and without loss of generality assume $U_{n} \supseteq U_{n+1}$. Then

$$
M_{s}\left(U_{n}\right)=\lambda\left(U_{n}\right)^{-1} S\left(U_{n}\right) \leqq \lambda\left(U_{n}\right)^{-1} S\left(K_{n}\right)<\frac{1}{2} \lambda\left(K_{n}\right)^{-1} S\left(K_{n}\right)=\frac{1}{2} M_{s}\left(K_{n}\right) .
$$

Consequently $M_{S}\left(U_{n}\right)$ bounded below implies $M_{S}\left(K_{n}\right)$ bounded below. Therefore we need only verify (2) for $\left\{K_{n}\right\} \subset \mathscr{K}_{0}$. The fundamental inequality implies, upon fixing any $A=A_{0} \in \mathscr{K}_{+}$,

$$
\begin{aligned}
\lambda\left(A_{0}\right)^{-1} S\left(K_{1} A_{0}\right) & \leqq \lambda\left(A_{0}\right)^{-1} S\left(K_{n} A_{0}\right) \leqq \lambda\left(K_{n}^{-1}\right)^{-1} S\left(K_{n}\right) \\
& \leqq C \lambda\left(K_{n}\right)^{-1} S\left(K_{n}\right)=C M_{S}\left(K_{n}\right)
\end{aligned}
$$

for some $C>0$ independent of $n$ by the same argument as in the proof of (1). Thus $\lambda\left(A_{0}\right)^{-1} S\left(K_{1} A_{0}\right) / C \leqq \inf M_{S}\left(K_{n}\right)$, and we are done as the converse implication is trivial.

\section{Regular set functions $S$ and their relation to} summing sequences. The question arises as to how useful the Følner sequences (or nets) are with respect to averaging regular set functions. One might initially suspect that they would be rather successful but the following simple example shows otherwise:

EXAMPLE 3.1. It is readily verified that $S(E)=-\lambda\left(E^{0}\right)$ is regular where $E^{0}$ is the interior of $E$. Moreover, it is easy to see that $\inf \left\{M_{S}(K): K \in \mathscr{K}_{+}\right\}=\inf \left\{M_{S}(K): K \in \mathscr{K}_{0} \cap \mathscr{K}_{c}\right\}=-1$ and $S$ is continu- 
ous at $\varnothing$. However, if we take $G=R$ and consider the strong summing sequence $\left\{A_{n}\right\}$ where

$$
A_{n}= \begin{cases}{[0, n],} & n \text { even } \\ {[0, n] \cap I,} & n \text { odd, where } I \text { denotes all irrational numbers. }\end{cases}
$$

Then $M_{S}\left(A_{n}\right)$ alternates between 0 and 1 and, of course, has no limit as $n \rightarrow+\infty$.

Consequently, even if $S$ is continuous at $\varnothing$, strong Følner summing sequences need not average $S$. This appears to be since $S$ may be rather "discontinuous" with respect to $\lambda$ whereas the Følner condition is not quite so sensitive. Nevertheless, in Theorem 3.3 we shall state conditions on $S$ which are sufficient for the utilization of Følner-like sequences in the averaging of $S$. For technical simplicity we assume $G$ is $\sigma$-compact and consequently consider sequences rather than nets [2, Theorem 4 and Proposition 1]. Before proceeding to the theorem we need the following:

Lemma 3.2. (i) If $K$ and $A$ are in $\mathscr{K}_{+}, \delta>0$, and

$$
\lambda(K A \Delta A)<\alpha \lambda(A),
$$

there exists $A^{*}=A(\delta, \alpha) \in \mathscr{K}, A^{*} \subseteq A$ such that $\lambda(K a-A)<\delta$, for all $a \in A^{*}$ and $\lambda\left(A^{*}\right) \geqq \lambda(A)\left(1-(\alpha / \delta) \lambda\left(K^{-1}\right)\right)$.

(ii) Also, if $K \in \mathscr{K}_{+}$and $\delta>0$ are fixed and $\left\{A_{n}^{-1}\right\}$ is a (right) strong Følner summing sequence in the unimodular group $G$, there exists $\left\{A_{n}^{*}\right\} \subseteq \mathscr{K}$, $A_{n}^{*} \subseteq A_{n}$ such that $\lambda\left(A_{n}\right)^{-1} \lambda\left(A_{n}^{*}\right) \rightarrow 1$ and

$$
\lambda\left(K a-A_{n}\right)<\delta \text { for all } a \in A_{n}^{*} .
$$

Proof. Let $E=\{a \in A: \lambda(K a-A) \geqq \delta\} . E$ is clearly measurable and moreover

$$
\begin{aligned}
\int_{E} \lambda & (K a-A) d \lambda(a)=\int_{E}\left\{\int_{K A-A} \chi_{K a}(b) d \lambda(b)\right\} d \lambda(a) \\
& =\int_{K A-A}\left\{\int_{E} \chi_{K a}(b) d \lambda(a)\right\} d \lambda(b) \\
& =\int_{K A-A}\left\{\int_{E} \chi_{K^{-1} b}(a) d \lambda(a)\right\} d \lambda(b) \\
& \leqq \int_{K A-A} \lambda\left(K^{-1} b\right) d \lambda(b)=\lambda(K A-A) \lambda\left(K^{-1}\right) \leqq \lambda(K A \Delta A) \lambda\left(K^{-1}\right) \\
& <\alpha \lambda(A) \lambda\left(K^{-1}\right) .
\end{aligned}
$$


But clearly

$$
\int_{E} \lambda(K a-A) d \lambda(a) \geqq \int_{E} \delta d \lambda(a)=\delta \lambda(E),
$$

and consequently

$$
\lambda(E) \leqq(\alpha / \delta) \lambda\left(K^{-1}\right) \lambda(A)
$$

implying if $A^{*} \doteqdot A-E, \lambda(K a-A)<\delta$ for $a \in A^{*}$ and

$$
\lambda\left(A^{*}\right)=\lambda(A)-\lambda(E) \geqq\left(1-(\alpha / \delta) \lambda\left(K^{-1}\right)\right) \lambda(A),
$$

as needed. Statement (ii) follows upon taking $A=A_{n}$ and choosing $A^{*}=A_{n}^{*}$ as indicated in (i) since for any $K$ in $\mathscr{K}_{+}$

$$
\lambda\left(K A_{n} \Delta A_{n}\right)<\alpha_{n} \lambda\left(A_{n}\right)
$$

where $\alpha_{n} \rightarrow 0$ by inversion invariance and the defining property of $\left\{A_{n}^{-1}\right\}$ and consequently $\left.\lambda\left(A_{n}\right)^{-1} \lambda\left(A_{n}^{*}\right) \geqq\left(\alpha_{n} / \delta\right) \lambda\left(K^{-1}\right)\right) \rightarrow 1$.

We are now ready to prove:

THEOREM 3.3. Let $G$ be unimodular and amenable, $S$ be a regular set function which is continuous at $\varnothing$ and upper continuous on $\mathscr{K}$, and $\left\{A_{n}\right\} \subseteq \mathscr{K}_{+}$satisfy $\lambda\left(A_{n}\right)^{-1} \lambda\left(g A_{n} \Delta A_{n}\right) \rightarrow 0$ for all $g \in G$ (i.e. $\left\{A_{n}^{-1}\right\}$ is a (right) weak Følner summing sequence in $G$ ). Then

$$
\lim M_{S}\left(A_{n}\right)=\inf \left\{M_{S}(K): K \in \mathscr{K}_{+}\right\}=\inf \left\{M_{S}(K): K \in \mathscr{K}_{0} \cap \mathscr{K}_{c}\right\} .
$$

Proof. The second equality follows from continuity at $\varnothing$. We first prove the result for a (right) strong Følner summing sequence $\left\{A_{n}^{-1}\right\}$ in $G$, i.e.

$$
\lambda\left(A_{n}\right)^{-1} \lambda\left(K A_{n} \Delta A_{n}\right)=\lambda\left(A_{n}^{-1}\right)^{-1} \lambda\left(A_{n}^{-1} K^{-1} \Delta A_{n}^{-1}\right) \rightarrow 0
$$

for all $K \in \mathscr{K}, K \neq \varnothing$, where unimodularity has been used to obtain inversion invariance of $\lambda$. Fix $K \in \mathscr{K}_{+}$and $\epsilon>0$. Since $S$ is upper continuous at $K$ by Proposition 1.5 (i) there is a $\delta=\delta(\epsilon, K)>0$ such that $K^{*} \in \mathscr{K}, K^{*} \subseteq K$, and $\lambda\left(K-K^{*}\right)<\delta$ implies $S\left(K^{*}\right)<S(K)+\epsilon$. Now for this $K$ and $\delta>0$, apply Lemma 3.2 (ii) to obtain the sequence $\left\{A_{n}^{*}\right\}$. First, since

$$
\lambda\left(K a-A_{n}\right)=\lambda\left(K a-\left(K a \cap A_{n}\right)\right)=\lambda\left(K-\left(K a \cap A_{n}\right) a^{-1}\right)<\delta
$$

for all $a \in A_{n}^{*}\left(\right.$ setting $\left.K^{*}=\left(K a \cap A_{n}\right) a^{-1}\right)$ we have

$$
S\left(K a \cap A_{n}\right)=S\left(\left(K a \cap A_{n}\right) a^{-1}\right)<S(K)+\epsilon .
$$


Next let $\left\{e_{n}\right\}$ be the sequence in Lemma 2.12 corresponding to $K$ and $A=A_{n}$. Moreover, in light of Lemma 2.9 and the proof of Lemma 2.12, the $\left\{e_{n}\right\}$ also may be chosen so that $\mu_{n}\left(A_{n}^{*}\right) \rightarrow \mu\left(A_{n}\right)=\lambda\left(A_{n}^{*}\right)^{-1} \lambda\left(A_{n}^{*}\right)$ (in the notation of Lemma 2.8). Following the proof of Proposition 2.13 (and using similar notation),

$$
\begin{aligned}
N^{-1} \sum_{j \leqq N} S\left(I_{j}\right) & \leqq N^{-1} \sum_{j \leqq N} S\left(K e_{j} \cap A_{n}\right) \\
& \leqq N^{-1} \sum_{j \leqq N, e_{j} \in A_{n}^{*}} S\left(K e_{j} \cap A_{n}\right) \leqq N^{-1} \sum_{j \leqq N, e_{j} \in A_{n}^{*}}(S(K)+\epsilon) \\
& =\mu_{N}\left(A_{n}^{*}\right)(S(K)+\epsilon),
\end{aligned}
$$

where

$$
\begin{aligned}
I_{j} & =\left\{g \in G: \sum_{i \leqq N} \chi_{K e_{i} \cap A_{n}}(g) \geqq j\right\} \\
& =\left\{a \in A_{n}: \sum_{i \leqq N} \chi_{K e_{i}}(a) \geqq j\right\} \\
& =\left\{a \in A_{n}: g_{N}(a) \geqq j / N\right\} \subseteq A_{n} .
\end{aligned}
$$

Consequently for any positive integer $j_{0}$,

$$
\left(j_{0} / N\right) S\left(A_{n}\right)+S\left(I_{j 0}\right) \leqq \mu_{n}\left(A_{n}^{*}\right)(S(K)+\epsilon) .
$$

Now fix $\rho>0$ and choose $j_{0}=\left[N\left(\lambda\left(A_{n}\right)^{-1} \lambda(K)+\rho\right)\right]$. Upon letting $N \rightarrow+\infty$ and reasoning as at the end of the proof of Proposition 2.13 we obtain

$$
\left(\lambda\left(A_{n}\right)^{-1} \lambda(K)+\rho\right) S\left(A_{n}\right) \leqq \lambda\left(A_{n}\right)^{-1} \lambda\left(A_{n}^{*}\right)(S(K)+\epsilon) .
$$

Next letting $\rho \downarrow 0$ first and then $n \rightarrow+\infty$ we finally obtain (since $\left.\lambda\left(A_{n}\right)^{-1} \lambda\left(A_{n}^{*}\right) \rightarrow 1\right)$

$$
\left(\varlimsup_{n} \lambda\left(A_{n}\right)^{-1} S\left(A_{n}\right)\right) \lambda(K)<S(K)+\epsilon,
$$

and since the left side does not depend on $\epsilon$,

$$
\varlimsup_{n} M_{S}\left(A_{n}\right) \leqq \lambda(K)^{-1} S(K)=M_{S}(K) \text { for all } K \in \mathscr{K}_{+} \text {, }
$$

and consequently,

$\lim _{n} M_{S}\left(A_{n}\right)=\inf \left\{M_{S}(K): K \in \mathscr{K}_{+}\right\}=\inf \left\{M_{S}(K): K \in \mathscr{K}_{0} \cap \mathscr{K}_{c}\right\}$ 
and the theorem is proved if $\left\{A_{n}^{-1}\right\}$ is strong Følner. However, if $\left\{A_{n}^{-1}\right\}$ is weak Følner by [2, Theorem 15] there exist $A_{n}^{*} \subseteq A_{n}$ such that

$$
\lambda\left(A_{n}\right)^{-1} \lambda\left(A_{n}^{*}\right)=\lambda\left(A_{n}^{-1}\right)^{-1} \lambda\left(A_{n}^{*-1}\right) \rightarrow 1
$$

and $\left\{A_{n}^{*-1}\right\}$ is strong Følner. Now $S\left(A_{n}\right) \leqq S\left(A_{n}^{*}\right)$ implies

$$
\begin{aligned}
M_{S}\left(A_{n}\right) & =\lambda\left(A_{n}\right)^{-1} S\left(A_{n}\right) \leqq \lambda\left(A_{n}\right)^{-1} S\left(A_{n}^{*}\right) \\
& =\left(\lambda\left(A_{n}^{*}\right)^{-1} S\left(A_{n}^{*}\right)\right)\left(\lambda\left(A_{n}\right)^{-1} \lambda\left(A_{n}^{*}\right)\right) \\
& =M_{S}\left(A_{n}^{*}\right)\left(\lambda\left(A_{n}\right)^{-1} \lambda\left(A_{n}^{*}\right)\right),
\end{aligned}
$$

and consequently,

$$
\varlimsup M_{S}\left(A_{n}\right) \leqq \lim M_{S}\left(A_{n}^{*}\right)
$$

which equals $\inf \left\{M_{S}(K): K \in \mathscr{K}_{+}\right\}$as we have already shown and the theorem is proved in general.

Comment. Lemma 3.2 and Theorem 3.3 were proved assuming implicitly that $K A$ is measurable. Otherwise, as by our convention, $\lambda$ denotes inner measure and straightforward modifications of the proofs cover this contingency.

\section{REFERENCES}

1. S. Banach, Sur le problème de mésure, Fund. Math., 4 (1923), 7-33.

2. W. R. Emerson, Ratio properties in locally compact amenable groups, Trans. Amer. Math. Soc., 133 (1968), 179-204.

3. _ - Averaging strongly subadditive set functions in unimodular amenable groups I, Pacific J. Math., 61 (1975), 391-400.

Received October 16, 1975 and in revised form January 30, 1976. Partially supported by NSF Grant GP-42920.

Queen's College (CUNY) 


\section{PACIFIC JOURNAL OF MATHEMATICS}

\section{EDITORS}

RICHARD ARENS (Managing Editor)

University of California

Los Angeles, California 90024

\author{
R. A. Beaumont \\ University of Washington \\ Seattle, Washington 98105
}

\section{J. DugunduI}

Department of Mathematics University of Southern California Los Angeles, California 90007

D. Gilbarg and J. Milgram

Stanford University

Stanford, California 94305

\section{ASSOCIATE EDITORS}

E. F. BECKENBACH
B. H. NeumanN

F. Wolp
K. YoshidA

\section{SUPPORTING INSTITUTIONS}

UNIVERSITY OF BRITISH COLUMBIA

CALIFORNIA INSTITUTE OF TECHNOLOGY

UNIVERSITY OF CALIFORNIA

MONTANA STATE UNIVERSITY

UNIVERSITY OF NEVADA

NEW MEXICO STATE UNIVERSITY

OREGON STATE UNIVERSITY

UNIVERSITY OF OREGON

OSAKA UNIVERSITY

\author{
UNIVERSITY OF SOUTHERN CALIFORNIA \\ STANFORD UNIVERSITY \\ UNIVERSITY OF HAWAII \\ UNIVERSITY OF TOKYO \\ UNIVERSITY OF UTAH \\ WASHINGTON STATE UNIVERSITY \\ UNIVERSITY OF WASHINGTON \\ AMERICAN MATHEMATICAL SOCIETY
}

The Supporting Institutions listed above contribute to the cost of publication of this Journal, but they are not owners or publishers and have no responsibility for its contents or policies.

Mathematical papers intended for publication in the Pacific Journal of Mathematics should be in typed form or offset-reproduced (not dittoed). double spaced with large margins. Underline Greek letters in red, German in green, and script in blue. The first paragraph or two must be capable of being used separately as a synopsis of the entire paper. Items of the biblography should not be cited there unless absolutely necessary, in which case they must be identified by author and Journal, rather than by item number. Manuscripts, in duplicate, may be sent to any one of the four editors. Please classify according to the scheme of Math. Reviews, Index to Vol. 39. All other communications should be addressed to the managing editor, or Elaine Barth, University of California, Los Angeles, California, 90024.

100 reprints are provided free for each article, only if page charges have been substantially paid. Additional copies may be obtained at cost in multiples of 50 .

The Pacific Journal of Mathematics is issued monthly as of January 1966. Regular subscription rate: $\$ 72.00$ a year (6 Vols., 12 issues). Special rate: $\$ 36.00$ a year to individual members of supporting institutions.

Subscriptions, orders for back numbers, and changes of address should be sent to Pacific Journal of Mathematics, 103 Highland Boulevard, Berkeley, California, 94708.

PUBLISHED BY PACIFIC JOURNAL OF MATHEMATICS, A NON-PROFIT CORPORATION Printed at Jerusalem Academic Press, POB 2390, Jerusalem, Israel.

Copyright (C) 1976 Pacific Journal of Mathematics All Rights Reserved 


\section{Pacific Journal of Mathematics}

\section{Vol. 64, No. $2 \quad$ June, 1976}

Richard Fairbanks Arnold and A. P. Morse, Plus and times............. 297

Edwin Ogilvie Buchman and F. A. Valentine, External visibility ......... 333

R. A. Czerwinski, Bonded quadratic division algebras.............. 341

William Richard Emerson, Averaging strongly subadditive set functions in unimodular amenable groups. II .........................

Lynn Harry Erbe, Existence of oscillatory solutions and asymptotic behavior for a class of third order linear differential equations ............. 369

Kenneth R. Goodearl, Power-cancellation of groups and modules........ 387

J. C. Hankins and Roy Martin Rakestraw, The extremal structure of locally compact convex sets ...................................

Burrell Washington Helton, The solution of a Stieltjes-Volterra integral

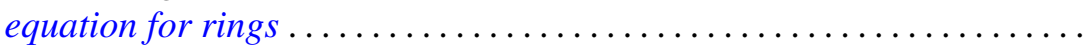

Frank Kwang-Ming Hwang and Shen Lin, Construction of 2-balanced

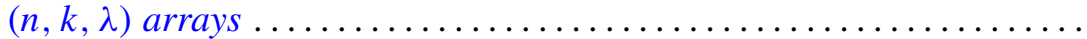

Wei-Eihn Kuan, Some results on normality of a graded ring ... 455

Dieter Landers and Lothar Rogge, Relations between convergence of series and convergence of sequences ......................... 465

Lawrence Louis Larmore and Robert David Rigdon, Enumerating immersions and embeddings of projective spaces ................

Douglas C. McMahon, On the role of an abelian phase group in relativized problems in topological dynamics..................

Robert Wilmer Miller, Finitely generated projective modules and TTF classes...

Yashaswini Deval Mittal, A class of isotropic covariance functions ...

Anthony G. Mucci, Another martingale convergence theorem ...

Joan Kathryn Plastiras, Quasitriangular operator algebras ...

John Robert Quine, Jr., The geometry of $p\left(S^{1}\right) \ldots \ldots \ldots$. 